\title{
Use of scaling relations to extract intrinsic fluorescence lifetime of targets embedded in turbid media
}

\author{
Victor Chernomordik \\ Moinuddin Hassan \\ Franck Amyot \\ Jason Riley \\ Amir Gandjbakhche \\ National Institutes of Health \\ National Institute of Child Health and Human \\ Development \\ Bethesda, Maryland 20892
}

\begin{abstract}
We present a novel method for estimating the intrinsic fluorescence lifetime of deeply embedded localized fluorophores. It is based on scaling relations, characteristic for turbid media. The approach is experimentally substantiated by successfully reconstructing lifetimes for targets at depths up to $14.5 \mathrm{~mm}$. A derived correction factor was determined from the product of the transport-corrected scattering coefficient $\mu_{s}^{\prime}$ and the index of refraction $n_{r}$. In addition, data from an array of detectors $(\geq 2)$ can be used to estimate $\mu_{s}^{\prime} n_{r}$. The suggested algorithm is a promising tool for diagnostic fluorescence, since lifetime can be a sensitive indicator of the fluorophore environment. () 2008 Society of Photo-Optical Instrumentation Engineers. [DOI: 10.1117/1.2904660]
\end{abstract}

Keywords: biomedical optics; fluorescence; light propagation; biological tissues.

Paper 07449LR received Nov. 5, 2007; revised manuscript received Jan. 2, 2008; accepted for publication Jan. 19, 2008; published online Apr. 17, 2008.
Due to recent advances in the development of molecular probes, fluorescence is increasingly being used to analyze biological processes at the molecular and cellular levels. The high specificity of fluorescence imaging explains the considerable interest in this modality by the biomedical community. Fluorescent markers conjugated with corresponding antibodies can target diseased cells, creating a promising tool for medical diagnostics. However, existing applications of fluorescence in biology and medicine are mainly limited to surface and/or thin tissue sample imaging. ${ }^{1,2}$ Advances in new fluorescent agents with higher quantum yields, operating in the near-IR spectral window, are favorable for the penetration of light into biological tissue and have stimulated new efforts in fluorescent imaging of deeply embedded targets. To make this modality quantitative, photon migration models that take into account the turbid nature of biological tissues should be developed. $^{3-5}$

Optical imaging data can be collected by three schemes: continuous wave (cw), time domain, or frequency domain. To choose the most suitable system, the trade-off between the cost of the system and measurable information should be taken into account. Low-cost cw imaging enables one to measure only 2-D intensity distributions of fluorescent light that can be used to map distributions of the fluorophores, while frequency-domain and especially time-domain measurements can provide much richer informational content, in particular, related to fluorescence lifetime distributions. Fluorescence lifetime information can be used to track possible alterations of physiological parameters (e.g., $\mathrm{pH}$ and temperature) resulting from malignant transformation of healthy tissue, because time-resolved spectroscopy of some specifically targeted fluorescent labeled antibodies is known to be sensitive to such variations. Changes in the physiological status of tissues can

Address all correspondence to Victor V. Chernomordik, NICHD, National Inst. of Health, Bldg. \#9 Rm. \#B1E11-Bldg. 9 Rm. B1E01, Bethesda, MD 20892; Tel: 301435 9236; E-mail: vchern@helix.nih.gov be observed by comparing these parameters inside an abnormality (or very close to it) to that of surrounding healthy tissue. Thus, a high-resolution functional image of the region of interest can be provided, which is important for early cancer diagnostics.

To take advantage of these rapidly improving experimental techniques, adequate inverse algorithms to reconstruct spatial and temporal characteristics of the deeply embedded fluorophores are required. The general inverse problem of diffuse optical tomography, including fluorescence tomography, is illposed, making it computationally expensive while reducing the accuracy of quantifying the optical parameters. For this reason, alternative approaches have been discussed in the literature. ${ }^{4}$ In general, they use analytical formulas obtained from photon migration models to derive lifetimes of deeply embedded targets from the time-of-flight distributions of fluorescent photons. The random walk model ${ }^{6,7}$ was applied to acquire closed-form expressions for deeply embedded fluorophores with relatively short lifetimes, when the time delay of photons between source and detector due to scattering was higher than the fluorescence lifetime. Kumar et al., ${ }^{4}$ considering an opposite case of a lifetime longer than the diffusive time scale, presented conditions under which the asymptotic behavior of emitted intensity reveals the intrinsic lifetime of the fluorophore. More recently, a theoretical analysis elucidated the diffuse and pure fluorescent decay components of diffuse time-resolved fluorescence. ${ }^{3}$ Using the Born approximation to the transport equation, the authors derived more general expressions for time-resolved fluorescence tomography, including the case of a low-absorbing medium, when the asymptotic approach fails and the entire time-resolved distribution should be used. The authors verified their approach with tomographic reconstruction of simulated data. However, the low signal-to-noise ratio (SNR) that is expected in the

$1083-3668 / 2008 / 13(2) / 024025 / 4 / \$ 25.00$ @ 2008 SPIE 
long-time tail of the distribution is potentially a problem for data analysis.

The goal of this study was to find and experimentally verify scaling relations that could be used to correct observed time-resolved intensity distributions from fluorescent targets at a given depth $z$ inside a turbid medium to an expected surface distribution (from the same fluorophore, but at $z=0$ ), revealing the intrinsic fluorescence lifetime without the necessity for full-scale reconstruction. Similar corrections could be applied when comparing the time-resolved data obtained from the same deeply embedded fluorophore by several detectors positioned at different distances from the source (excitation photon entry point into the medium). This research focuses on the shape of the corresponding time-resolved curve, important lifetime estimates, not on amplitude scaling.

The basis for this approach is a diffusion-like photon migration model, either the random walk theory or the diffusion equation. According to these models, the dependence of timeresolved reflectance on the source-detector separations $r$ is limited to an exponential factor $\exp \left[-\left(3 \mu_{s}^{\prime} n_{r} r^{2} / 4 c t\right)\right]$, where $t$ is the photon time of flight, and $c$ is the speed of light in a vacuum (see, e.g., Ref. 8). Thus, the ratio of reflected intensities for source-detector separations $r$ and zero is

$$
\frac{\Gamma_{e}(t, r)}{\Gamma_{e}(t, 0)}=\exp \left(-\frac{3 \mu_{s}^{\prime} n_{r} r^{2}}{4 c t}\right) .
$$

Strictly speaking, Eq. (1) implies the same background optical parameters (scattering and absorption) of the medium at excitation and emission wavelengths $\lambda_{\mathrm{ex}}$ and $\lambda_{\mathrm{em}}$. Under this condition the ratio $\Gamma_{e}(t, r) / \Gamma_{e}(t, 0)$ does not depend on absorption because for the same time of flight, it results in the same attenuation in the numerator and denominator.

Making the preceding assumption [and Eq. (1)] is justified if the selected values of $\lambda_{\mathrm{ex}}$ and $\lambda_{\mathrm{em}}$ are close and provide nonsignificant absorption variations in the medium (scattering coefficient changes), described by a power law, and are expected to be small for $\left(\lambda_{\mathrm{em}}-\lambda_{\mathrm{ex}}\right) / \lambda_{\mathrm{ex}} \ll 1$. Practically, one can use the average value of scattering coefficient $\mu_{s}^{\prime}$ $=\left(\mu_{s, \mathrm{ex}}^{\prime}+\mu_{s, \mathrm{em}}^{\prime}\right) / 2$ in Eq. (1).

In an isotropic medium with the same-source-detector geometry, we can expect a similar relationship between timeresolved fluorescence curves from a surface fluorophore and one embedded at depth $z$ :

$$
\frac{\Gamma_{e}(t, z)}{\Gamma_{e}(t, 0)}=\exp \left(-\frac{3 \mu_{s}^{\prime} n_{r} z^{2}}{c t}\right) .
$$

The factor 4 in the denominator of the exponent index is absent because a photon travels the distance $z$ twice (first as an excitation photon and then as an emission photon to the detector).

To verify the introduced correction factors, we used a recently developed time-resolved imaging system (details are given in Ref. 9). Data are collected by 2-D scanning of the region of interest (ROI) by a linear fiber array with fixed separations between a single source fiber and several detection fibers. The impulse response function (IRF) width in our experiments was $0.35 \mathrm{~ns}$. Our simulations show that it may introduce an error of $<1 \%$ into estimates of fluorescence life-

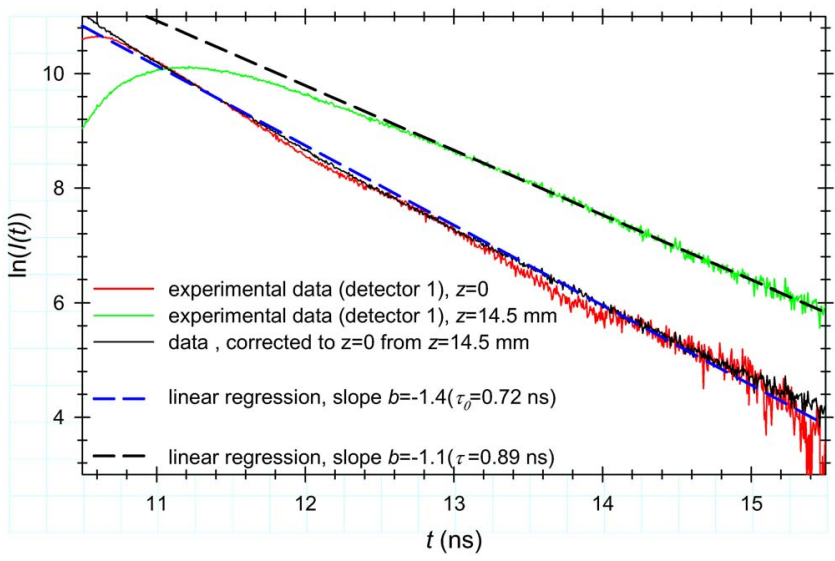

Fig. 1 Time-resolved intensities of two fluorophores with depths $z$. The corrected data show the closeness of the reconstructed lifetime to the intrinsic lifetime.

time $(\tau>0.7 \mathrm{~ns})$. To assess the performance of the instrument, tissue-like highly scattering phantoms were created with a fluorescent target placed at different depths inside the medium. These phantoms were prepared from an agarosebased gel, containing a small target microbeads of Alexa Fluor 750 (Invitrogen Inc., California, USA) in water solution. The fluorescence excitation/emission wavelengths were $750 / 790 \mathrm{~nm}$. Total absorption coefficients of many human tissues in vivo (e.g., breast) are close at these wavelengths ${ }^{10}$ $\left(2\left|\mu_{a}\right|_{750 \mathrm{~nm}}-\left.\mu_{a}\right|_{790 \mathrm{~nm}} \mid\right) /\left(\left.\mu_{a}\right|_{750 \mathrm{~nm}}+\left.\mu_{a}\right|_{790 \mathrm{~nm}}\right) \leq 10 \%$, justifying our approach for further biomedical applications of similar dyes.

To simulate the scattering properties of tissues, $10 \%$ Intralipid solution was added to the phantom gel for a final lipid concentration of $0.85 \%$. Based on the literature, expected values $^{11,12}$ were $n_{r} \approx 1.4$ and $\mu_{s}^{\prime} \approx 2.1(0.85 / 2) \mathrm{mm}^{-1}$ $\approx 0.89 \mathrm{~mm}^{-1}$ for $\lambda=780 \mathrm{~nm}$. Phantom dimensions were sufficient to provide semiinfinite geometry with respect to the fluorescent target. The fluorophore depth $z$ was varied by adding slabs of phantom material placed above the original surface. A zero time shift $t_{0} \approx 9.9 \mathrm{~ns}$ was introduced in the experimental setup to center the observed curves in the 25-ns measurement window. The experimental setup used for the model verification is very basic: the source fiber at the sample surface and two detection fibers at given distances from the source (source-detector separations $r_{1}$ and $r_{2}$, respectively). For simplicity, we considered experimental data with the excitation source fiber placed directly above a single fluorophore, though our general conclusions concerning scaling relations in Eqs. (1) and (2) do not change.

A series of experiments with different target depths $z=0$ to $14.5 \mathrm{~mm}$ clearly demonstrated the dependence of the apparent fluorescence lifetime-determined as an observed exponential decay time of emission intensity $I(t): \tau=-\{\mathrm{d} \ln [I(t)] / \mathrm{d} t\}^{-1}$-on the fluorophore depth. Due to light diffusion deeper embedded fluorophores showed longer apparent lifetimes (not to be confused with intrinsic fluorescence lifetime, determined by the fluorophore itself and its immediate environment).

Time-resolved data from a fluorophore at $z=0,14.5 \mathrm{~mm}$ are presented in Fig. 1. The difference in the slopes of long 


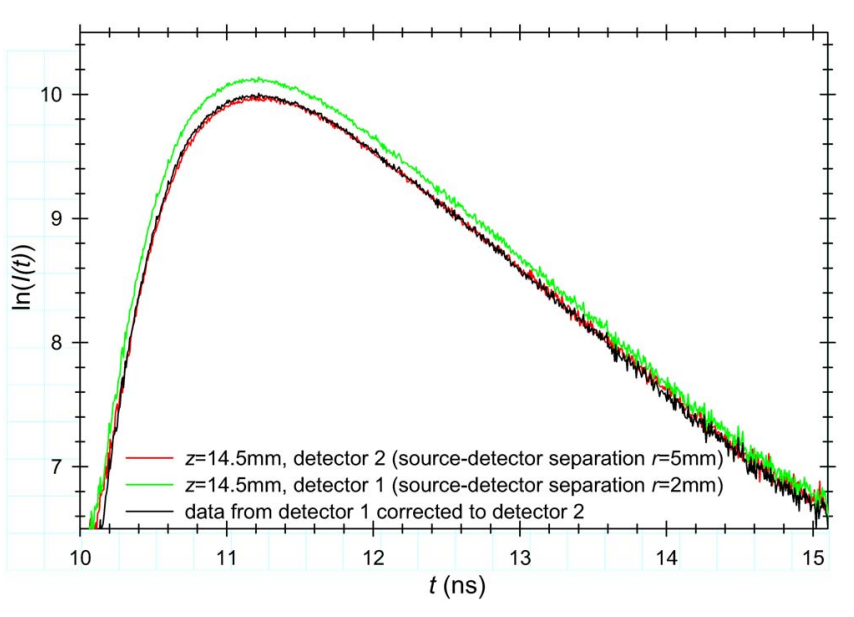

Fig. 2 Time-resolved data obtained from the fluorophore by detectors with source-detector separations of 2 and $5 \mathrm{~mm}$, respectively.

time tails of the two distributions can be clearly seen (compare with simple asymptotic model of Ref. 4, where similar slopes were expected). This corresponds to observed lifetimes of $\tau_{0} \approx 0.72 \mathrm{~ns}$ for $z=0 \mathrm{~mm}$ and $\tau \approx 0.89 \mathrm{~ns}$ for $z=14.5 \mathrm{~mm}$. For comparison note that the lifetime of Alexa Fluor 750 in water solution reported by the manufacturer (http://probes.invitrogen.com/servlets/datatable? $\mathrm{id}=38089$ ) is $\tau_{n} \approx 0.7 \mathrm{~ns} \approx \tau_{0}$ (our measurements gave $\tau_{n} \approx 0.68 \mathrm{~ns}$ ). Using Eq. (2) with known $\mu_{s}^{\prime} \approx 0.9 \mathrm{~mm}^{-1}$ and $n_{r} \approx 1.4$ to modify the measured distribution for $z=14.5 \mathrm{~mm}$, we can compare the expected distribution at the sample surface with the observed value. Excluding amplitude scaling, which can be seen as a constant shift in logarithmic scale, the agreement is good. In the broad time range $10.5 \mathrm{~ns} \leq t \leq 15.5 \mathrm{~ns}$ both dependencies $\ln [I(t)]$ are close to linear (linear regression correlation coefficient $\left.r^{2} \simeq 0.99\right)$ with practically the same slopes $(1.39$ versus $\left.1.41 \mathrm{~ns}^{-1}\right)$. This fact demonstrates the possibility to estimate intrinsic lifetime from the time-resolved data obtained from a deeply embedded fluorophore. Similar results were obtained for several other depths $z=6$ to $13.8 \mathrm{~mm}$ (data not shown).

A comparison of the data collected from the same fluorophore at $z=14.5 \mathrm{~mm}$ by different fibers further substantiates our model. Figure 2 presents time-resolved distributions, measured by two fibers at source-detector separations $r_{1}$ and $r_{2}$ of 2 and $5 \mathrm{~mm}$. Transformation of data from the first detector, according to Eq. (1) with the same $\mu_{s}^{\prime}$ and $n_{r}$ but different source-detector separation $r_{2}$ predicts the expected shape of the distribution at the second detector. The discrepancy between the predicted and measured distributions (excluding constant amplitude scaling) is small $(<5 \%)$ over the broad range $10.5 \mathrm{~ns} \leq t \leq 15.5 \mathrm{~ns}$.

If two time-resolved data sets from the same target at different positions inside the medium are available, they can be used to estimate the product $\mu_{s}^{\prime} n_{r}$ from Eqs. (1) and (2). For example, by analyzing the linear regression of $\ln \left[\left.I(t)\right|_{z=0} /\left.I(t)\right|_{z=14.5 \mathrm{~mm}}\right]$ relative to $1 /\left(t-t_{0}\right)$, one can find the slope $\left(3 z^{2} / c\right) \mu_{s}^{\prime} n_{r} \approx 2.66$, corresponding to $\mu_{s}^{\prime}$ $\approx 0.9 \mathrm{~mm}^{-1}$, if $n_{r}=1.4$ (see Fig. 3). A similar estimate of $\mu_{s}^{\prime}$, but with greater error due to smaller difference in distances,

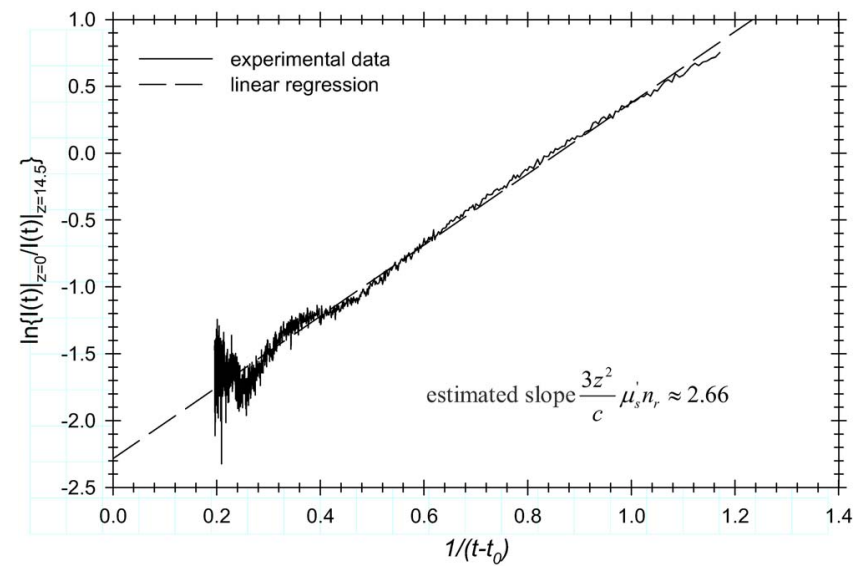

Fig. 3 Linear regression of intensities ratio (in logarithmic scale) for two depths as a function of $1 /\left(t-t_{0}\right)$.

can be obtained by comparing signals at two detectors (not presented).

The results of our analysis show that the simple scaling relationships of Eqs. (1) and (2) enable one to estimate the intrinsic fluorescence lifetime of a deeply embedded small fluorophore without full-scale reconstruction if the product $\mu_{s}^{\prime} n_{r}$ as well as the relative positions of the source, target, and detector are known. We can estimate $\mu_{s}^{\prime} n_{r}$ if two data sets with known geometrical setups are available (the simplest case is the same fluorophore and two detectors with significantly different source-detector separations). As for the fluorophore position, it can be estimated independently, opening the way for lifetime reconstruction. Lateral coordinates can be found by scanning the sample surface to search for the maximum emitted intensity (e.g., Ref. 13). Several algorithms to estimate target depth have been suggested in the literature: for time-resolved data, considering position of the fluorescence temporal point-spread function (TPSF) maximum ${ }^{14}$ and the mean time of the fluorescent curves, ${ }^{15}$ and for cw data analyzing the width of 2-D intensity distribution. ${ }^{13,16}$

In summary, we suggest a multistep analysis of timeresolved data on deeply embedded fluorescent targets to estimate intrinsic fluorescence lifetime, avoiding the necessity to solve an ill-posed inverse problem:

1. Localize the target in three dimensions. The lateral coordinates correspond to a maximum intensity of emitted light, found by scanning of the region of interest; the depth is determined either from the maximum of the TPSF, the mean time of flight, or cw intensity distributions.

2. Analyze data obtained from several source-detector separations, using Eq. (1) to estimate $\mu_{s}^{\prime} n_{r}$ (as was recently presented in Ref. 12) and compare results for several sourcedetector pairs, verifying the validity of medium uniformity assumption.

3. Introduce the correction of Eq. (2) into the observed lifetime intensity distribution to determine the intrinsic fluorescence lifetime from the obtained "surface" intensity distribution.

The accuracy of our approach depends on the accuracy of 3-D localization of the fluorophore, and to a lesser extent, on an error in our preliminary knowledge of $\mu_{s}^{\prime} n_{r}$. Since the 
relative positions of the source and detector are given, and the fluorophore lateral coordinates are estimated straightforwardly from the surface intensity distribution (e.g., Ref. 17), the main concern is the sensitivity of the lifetime estimate to assumed fluorophore depth. According to Refs. 15-17, expected errors in the depth are $\leq 10 \%$. To simulate potential errors in depth estimate, we replaced the genuine $z$ value of $z=14.5 \mathrm{~mm}$ by $z=13.2$ or $16 \mathrm{~mm}$, introducing the $10 \%$ depth error into our analysis. After modified correction, the emission intensity distribution reveals lifetimes of 769 and 662 ns, respectively, instead of $719 \mathrm{~ns}$, corresponding to real $z$ $=14.5 \mathrm{~mm}$ (deviation of $\sim 7$ to $8 \%$ ). Note that the lifetime estimate from the noncorrected data of $885 \mathrm{~ns}$ is much higher $(\sim 23 \%)$. Note that for the suggested correction factor, an error of $20 \%$ in $\mu_{s}^{\prime} n_{r}$ is equivalent to an error $\sim 10 \%$ in $z$.

In the case of several fluorescent targets, we should first localize them (in the case of two fluorophores in the ROI, this can be done, for example, by curve fitting of cw data, as described in Refs. 13 and 17). For the case of the fluorescent layers considered, for example, in Ref. 5 the necessary corrections to the observed decay time of emitted light can be found by integrating Eqs. (1) and (2) over lateral coordinates of the fluorophore $(x, y)$.

Note that although this paper discusses reflectance geometry, a similar approach using basic scaling relations for photon migration should be applicable for transmission geometry. We are currently considering its applications for small animal fluorescent imaging.

\section{Acknowledgment}

The authors acknowledge funding by the Intramural Research Program of National Institute of Child Health and Human Development of the National Institutes of Health.

\section{References}

1. S. Achilefu, C. H. Contag, A. P. Savitsky, and R. Weissleder, "Chemical and genetic sensors in biomedical research," J. Biomed. Opt. 10, 041201 (2005).

2. V. Ntziachristos, "Fluorescence molecular imaging," Аnnu. Rev. Biomed. Eng. 8, 1-33 (2006).

3. A. T. N. Kumar, S. B. Raymond, G. Boverman, D. A. Boas, and B. J. Bacskai, "Time resolved fluorescence tomography of turbid media based on lifetime contrast," Opt. Express 14, 12255-12270 (2006).
4. A. T. N. Kumar, J. Skoch, B. J. Bacskai, D. A. Boas, and A. K. Dunn, "Fluorescence-lifetime-based tomography for turbid media," Opt. Lett. 30, 3347-3349 (2005).

5. M. Chandra, K. Vishwanath, G. D. Fichter, E. Liao, S. J. Hollister, and M. A. Mycek, "Quantitative molecular sensing in biological tissues: an approach to noninvasive optical characterization," Opt. Express 14, 6157-6171 (2006).

6. D. Hattery, V. Chernomordik, M. Loew, I. Gannot, and A. Gandjbakhche, "Analytical solutions for time-resolved fluorescence lifetime imaging in a turbid medium such as tissue," J. Opt. Soc. Am. A Opt. Image Sci. Vis 18, 1523-1530 (2001).

7. I. Gannot, I. Ron, F. Hekmat, V. Chernomordik, and A. Gandjbakhche, "Functional optical detection based on $\mathrm{pH}$ dependent fluorescence lifetime," Lasers Surg. Med. 35, 342-348 (2004).

8. R. Cubeddu, A. Pifferi, P. Taroni, A. Torricelli, and G. Valentini, "Experimental test of theoretical models for time-resolved reflectance," Med. Phys. 23, 1625-1633 (1996).

9. M. Hassan, J. Riley, V. Chernomordik, P. Smith, R. Pursley, S. B. Lee, J. Capala, and A. H. Gandjbakhche, "Fluorescence lifetime imaging system for in vivo studies," Mol. Imaging 6, 229-236 (2007).

10. A. Pifferi, J. Swartling, E. Chikoidze, A. Torricelli, P. Taroni, A. Bassi, S. Andersson-Engels, and R. Cubeddu, "Spectroscopic timeresolved diffuse reflectance and transmittance measurements of the female breast at different interfiber distances," J. Biomed. Opt. 9(6), 1143-1151 (2004).

11. A. K. Dunn, V. P. Wallace, M. Coleno, M. W. Berns, and B. J. Tromberg, "Influence of optical properties on two-photon fluorescence imaging in turbid samples," Appl. Opt. 39, 1194-1201 (2000).

12. L. Spinelli, F. Martelli, A. Farina, A. Pifferi, A. Torricelli, R. Cubeddu, and G. Zaccanti, "Calibration of scattering and absorption properties of a liquid diffusive medium at NIR wavelengths. Timeresolved method," Opt. Express 15, 6589-6604 (2007).

13. A. Eidsath, V. Chernomordik, A. Gandjbakhche, P. Smith, and A. Russo, "Three-dimensional localization of fluorescent masses deeply embedded in tissue," Phys. Med. Biol. 47, 4079-4092 (2002).

14. D. Hall, G. B. Ma, F. Lesage, and W. Yong, "Simple time-domain optical method for estimating the depth and concentration of a fluorescent inclusion in a turbid medium," Opt. Lett. 29, 2258-2260 (2004).

15. A. Laidevant, A. Da Silva, M. Berger, J. Boutet, J. M. Dinten, and A. C. Boccara, "Analytical method for localizing a fluorescent inclusion in a turbid medium," Appl. Opt. 46, 2131-2137 (2007).

16. C. D'Andrea, L. Spinelli, D. Comelli, G. Valentini, and R. Cubeddu, "Localization and quantification of fluorescent inclusions embedded in a turbid medium," Phys. Med. Biol. 50, 2313-2327 (2005).

17. V. Chernomordik, D. Hattery, I. Gannot, and A. H. Gandjbakhche, "Inverse method 3-D reconstruction of localized in vivo fluorescence-application to Sjogren syndrome," IEEE J. Sel. Top. Quantum Electron. 5, 930-935 (1999). 